OPEN ACCESS

Edited by:

Salvatore Piscuoglio,

University Hospital of

Basel, Switzerland

Reviewed by:

Wilma Mesker,

Leiden University Medical

Center, Netherlands

George S. Karagiannis,

Albert Einstein College of Medicine,

United States

*Correspondence:

Annika Blank

annika.blank@pathology.unibe.ch

tThese authors have contributed equally to this work

Specialty section:

This article was submitted to

Pathology,

a section of the journal

Frontiers in Medicine

Received: 25 August 2019 Accepted: 15 October 2019

Published: 31 October 2019

Citation:

Blank A, Schenker C, Dawson H, Beldi G, Zlobec I and Lugli A (2019) Evaluation of Tumor Budding in

Primary Colorectal Cancer and Corresponding Liver Metastases

Based on H\&E and Pancytokeratin

Staining. Front. Med. 6:247. doi: 10.3389/fmed.2019.00247

\section{Evaluation of Tumor Budding in Primary Colorectal Cancer and Corresponding Liver Metastases Based on H\&E and Pancytokeratin Staining}

\author{
Annika Blank ${ }^{1 * \dagger}$, Carla Schenker ${ }^{1 \dagger}$, Heather Dawson ${ }^{1}$, Guido Beldi ${ }^{2}$, Inti Zlobec ${ }^{1}$ and \\ Alessandro Lugli ${ }^{1}$ \\ ${ }^{1}$ Institute of Pathology, University of Bern, Bern, Switzerland, ${ }^{2}$ Department of Visceral Surgery and Medicine, Inselspital, Bern \\ University Hospital, University of Bern, Bern, Switzerland
}

In colorectal cancer, tumor budding is associated with tumor progression and represents an additional prognostic factor in the TNM classification. Tumor buds can be found at the invasive front (peritumoral budding; PTB) and in the tumor center (intratumoral budding; ITB) of primary tumors. Previous studies have shown that tumor buds are also present in colorectal liver metastases $(C R L M)$. Data on the prognostic and predictive role in this clinical context are still sparse and no standardized approach to evaluate budding in CRLM has been published so far. This study aimed to analyze and correlate perimetastatic $(\mathrm{PMB})$ and intrametastatic budding $(\mathrm{IMB})$ on $\mathrm{H} \& \mathrm{E}$ and pancytokeratin staining, compare it to budding results in corresponding primary tumors and to propose a standardized scoring system in CRLM as the basis for future studies. Tumor tissue of 81 primary tumors and 139 corresponding CRLM was used for ngTMA construction. For each primary tumor and metastasis, two punches from the center and two punches from the periphery from areas with highest tumor budding density were included. TMA slides were stained for H\&E and pancytokeratin (Pan-CK). PTB, ITB, PMB, and IMB were analyzed and classified as bd1, bd2, and bd3 according to ITBCC guidelines. ITB and PTB as well as IMB and PMB showed significant correlation on H\&E and Pan-CK staining. No correlation was found for tumor bud counts in primary tumors and corresponding metastases. The agreement for categorized tumor bud counts showed fair to good agreement for metastases and poor agreement for primary tumors between different classes on H\&E and Pan-CK staining. Based on our results, tumor budding in primary tumors and CRLM seems to be different processes which might be the results of differing surrounding microenvironments. The evaluation of tumor budding in CRLM is challenging in cases without desmoplastic stroma reaction or intense perimetastatic ductular reaction. We therefore propose to evaluate tumor budding only in metastases with desmoplastic stroma reaction based on H\&E staining since important morphological features are obscured on Pan-CK staining.

Keywords: tumor budding, metastasis, intratumoral budding, intrametastatic budding, peritumoral budding, perimetastatic budding 


\section{INTRODUCTION}

In colorectal cancer, tumor budding is associated with tumor progression, local and distant metastases (1) and is an additional prognostic factor in the TNM classification published by the UICC (2). In 2016, the international tumor budding consensus conference (ITBCC) proposed a standardized scoring system, validated by several studies over the last few years (3-12). Additionally, the ITBCC highlighted the importance of tumor budding especially in two clinical scenarios: in pT1 CRC, tumor budding may be an indicator of an oncologic resection and in stage II CRC of adjuvant therapy, respectively (13).

A geographic histological analysis of CRC revealed the presence of tumor buds not only at the invasive tumor front (peritumoral budding, PTB), but also within the main tumor body (intratumoral budding, ITB) (14). The clinical value of ITB is its potential assessment in preoperative rectal cancer biopsies (15) and the prognostic significance shown now by several studies $(16,17)$.

An additional clinical scenario for tumor budding may be the management of stage IV CRC. The treatment of colorectal cancer liver metastases (CRLM) includes surgery alone and/or a combination with systemic chemotherapy. In a recent study, tumor budding was analyzed on a monocentric patient cohort $(n=229)$ which underwent a first surgical resection of CRLM (18). Tumor budding was counted on H\&E slides using a quantitative method selecting the area with highest density and counting sequential HPFs and shown to be a prognostic factor in univariate, but not in multivariate analysis (18). Nevertheless, there is not enough data in the literature to make final conclusions on the prognostic or predictive value of tumor budding in CRLM.

One of the main lessons learnt from the ITBCC is the stepwise validation of promising histological biomarkers and their potential value in daily practice. Therefore, we embarked in this preliminary study with three well-defined aims: first, to systematically analyze the geographic map of tumor budding in CRLM by introducing two terms, namely intrametastatic budding (IMB) and perimetastatic budding $(\mathrm{PMB})$ and difficulties associated with the assessment of budding in hepatic resections; second, to score IMB and PMB on pancytokeratin (Pan-CK) and H\&E stained slides based on the ITBCC method; third, to propose a scoring system for tumor budding in CRLM as a basis for future large multi-centric retrospective and prospective studies.

\section{MATERIALS AND METHODS}

\section{Patient Cohort}

Histological slides from a retrospective cohort of initially 110 patients surgically treated between 2000 and 2016 at the Inselspital Bern for their primary CRC and synchronous or metachronous CRLM were screened for tumor budding. Tumors without tumor budding in either the primary CRC or corresponding CRLM were excluded from the cohort. The final cohort included 81 patients of which one patient had two metachronous primary CRC. Formalin-fixed paraffin-embedded tissues from 82 primary CRC and 139 corresponding CRLM were used for this study and their corresponding clinicopathological data are summarized in Table $\mathbf{1 .}$

\section{Slide Scanning and Annotations}

$\mathrm{H} \& \mathrm{E}$ slides of all cases were reviewed to identify tumor blocks from primary tumors and liver metastases with highest density of tumor buds at the tumor front and within the tumor. The tumor front was defined as the desmoplastic stroma surrounding the

TABLE 1 | Clinicopathological features.

Clinicopathological features $(N=81)$

Gender

Male

Female

Histological subtype (primary)

Adeno

Mucinous

Tumor location (primary)

Left

Right

Rectum

Rectosigmoid

pT

pT1

pT2

pT3

pT4

$\mathrm{pN}$

pNO

pN1-2

Tumor grade (primary)

G1-2

G3

Neoadjuvant therapy

Lymphatic invasion (primary)

LO

L1

Venous invasion (primary)

vo

V1

Perineural invasion (primary)

$\mathrm{PnO}$

Pn1

MMR status

Deficient

Proficient

Time to metastasis

Synchronous

Metachronous

Number of metastases

Median

Range 
most advancing parts of the main tumor body. Only resection specimens were considered for the study.

Selected tumor blocks were re-cut and slides were stained for H\&E. All H\&E stained slides were scanned (Pannoramic P250, 3D Histech, Hungary, 20 $\times$ objective lens) and uploaded onto a digital platform (http://ngtma.path.unibe.ch/casecenter). Digital slides were reviewed and areas with highest density of tumor budding were annotated using a TMA annotation tool (Panoramic viewer v15.1 and TMA annotation tool, 3D Histech, Hungary). Different colors for tumor front (blue color) and center (red color) were used. Two annotations from the tumor center and two annotations from the tumor front were placed onto the digital slides whenever possible.

\section{Next-Generation Tissue Microarray (ngTMA ${ }^{\circledR}$ ) Construction}

Eighty-two blocks from primary tumors and 144 blocks from liver metastases served as donor blocks for ngTMA construction. In CRLM with only few vital tumor cells it was necessary to include more than one tumor block. Donor blocks and annotated digital slides were loaded into an automated tissue microarrayer (Grandmaster, 3D Histech). An image of each donor block was taken and superimposed onto the digital slide for exact correspondence. After confirming each annotation, punches with a diameter of $1 \mathrm{~mm}$ from donor blocks were taken and transferred into a recipient block. A total of 328 punches from primary tumors (tumor front: 164; tumor center: 162) and a total number of 560 punches from liver metastases (tumor front: 284; tumor center: 276) were included in the ngTMA.

\section{Immunohistochemistry}

TMA blocks were sectioned at $2.5 \mu \mathrm{m}$. Sections were mounted on glass slides, dried and baked at $60^{\circ} \mathrm{C}$ for $30 \mathrm{~min}$. H\&E staining and double immunohistochemistry for Pan-CK and CD8 were performed. Double immunohistochemistry was performed using Bond RX (Leica Biosystems). Slides were dewaxed using Bond dewax solution (product code AR9222, Leica Biosystems). Heatinduced epitope retrieval in citrate buffer based (code AR9640, Leica Biosystems) at $\mathrm{pH} 6$ for $20 \mathrm{~min}$ at $100^{\circ} \mathrm{C}$ was followed by incubation with primary mouse pancytokeratin antibody (Dako, clone AE1/AE3, Ref M351501-2); dilution 1:400; for $30 \mathrm{~min}$. Slides were incubated with HRP (horseradish peroxidase)polymer for $15 \mathrm{~min}$. Visualization was accomplished using 3,3Diaminobenzidine $(\mathrm{DAB})$ for $10 \mathrm{~min}$, leading to a brown staining signal (Bond polymer refine detection, Leica Biosystems, Ref DS9800). As a second step, mouse CD8 antibody was used (DakoAgilent, clone C8/144B, Ref M7103); dilution 1:100; incubation time $30 \mathrm{~min}$. Alkaline Phosphatase (AP)-polymer was used as secondary antibody; incubation time $15 \mathrm{~min}$. Visualization was accomplished using fast red resulting in a red chromogen (Red polymer refine Detection, Leica Biosystems, Ref DS9390). Samples were counterstained with hematoxylin and mounted with Aquatex (Merck).

\section{Evaluation of H\&E and Immunohistochemistry}

All ngTMA slides were scanned (Pannoramic P250, 3DHistech, Hungary, $20 \times$ objective lens) and evaluated using Scorenado, a
TMA analysis tool for digital TMA slides, as described previously (19). Each tumor punch contained an area of $0.785 \mathrm{~mm}^{2}$. Only Pan-CK staining was used to evaluated tumor budding in the present study. Tumor buds were defined as single cells or cell cluster of up to 4 tumor cells according to the ITBCC guidelines (13). One experienced pathologist (A.L.) evaluated the number of tumor buds on H\&E and Pan-CK staining at the tumor front (PTB and PMB) as well as intratumoral (ITB and IMB). Representative examples for PMB and IMB are given in Figure 1.

\section{Statistical Analysis}

Descriptive statistics were performed to determine the number of tumor buds in both $\mathrm{H} \& \mathrm{E}$ and $\mathrm{CK}$, including mean, minimum and maximum values across the center and tumor front in both primary tumor and metastatic lesions. Pearson's correlation coefficient was used to determine the strength of linear association between budding counts. $P$-values $<0.05$ (twosided) were considered statistically significant. Kappa statistics and $95 \% \mathrm{CI}$ were used to investigate agreement in $\mathrm{BD}$ categories between primary and metastatic tumors. The agreement was again determined using intraclass correlation coefficients (ICC values) for raw budding counts. All analyses were performed using SAS v9.4, the SAS Institute (Cary, NC).

\section{RESULTS}

Tumor budding was assessed by counting all tumor buds per punch on H\&E and Pan-CK staining. For each patient the mean, minimum and maximum number of ITB, PTB, IMB, and PMB was recorded. Results are summarized in Table 2. A significant difference was found between the mean number of tumor buds in primary tumors and metastases on Pan-CK staining but not on H\&E (Table 3).

The correlation coefficients for ITB, PTB, IMB, and PMB on H\&E and Pan-CK staining are included in Table 4. ITB and PTB showed significant correlation on H\&E. IMB and PMB showed significant correlation on H\&E and Pan-CK staining. No correlation was detected for tumor bud counts in primary tumors and corresponding metastases except for IMB on H\&E staining in comparison to ITB on Pan-CK staining.

Tumor bud counts from primaries and metastases were categorized as bd1, bd2, and bd 3 according to the ITBCC guidelines (13) on H\&E as well as on Pan-CK. The agreement for categorized tumor bud counts were estimated using kappa values. For metastases there was fair to good agreement. For primary tumors, agreement was poor, between different classes on H\&E and Pan-CK staining. Results are given in Table 5.

\section{DISCUSSION}

The process of tumor budding in primary tumors and liver metastases seems to be different. Although there was an association between PTB and ITB as well as PMB and IMB, respectively, a correlation between tumor budding in the primary tumor and the corresponding metastases was not observed.

From a biological point of view, the present results could make sense based on the following hypothesis. The formation of tumor budding has been shown to be an important step in 


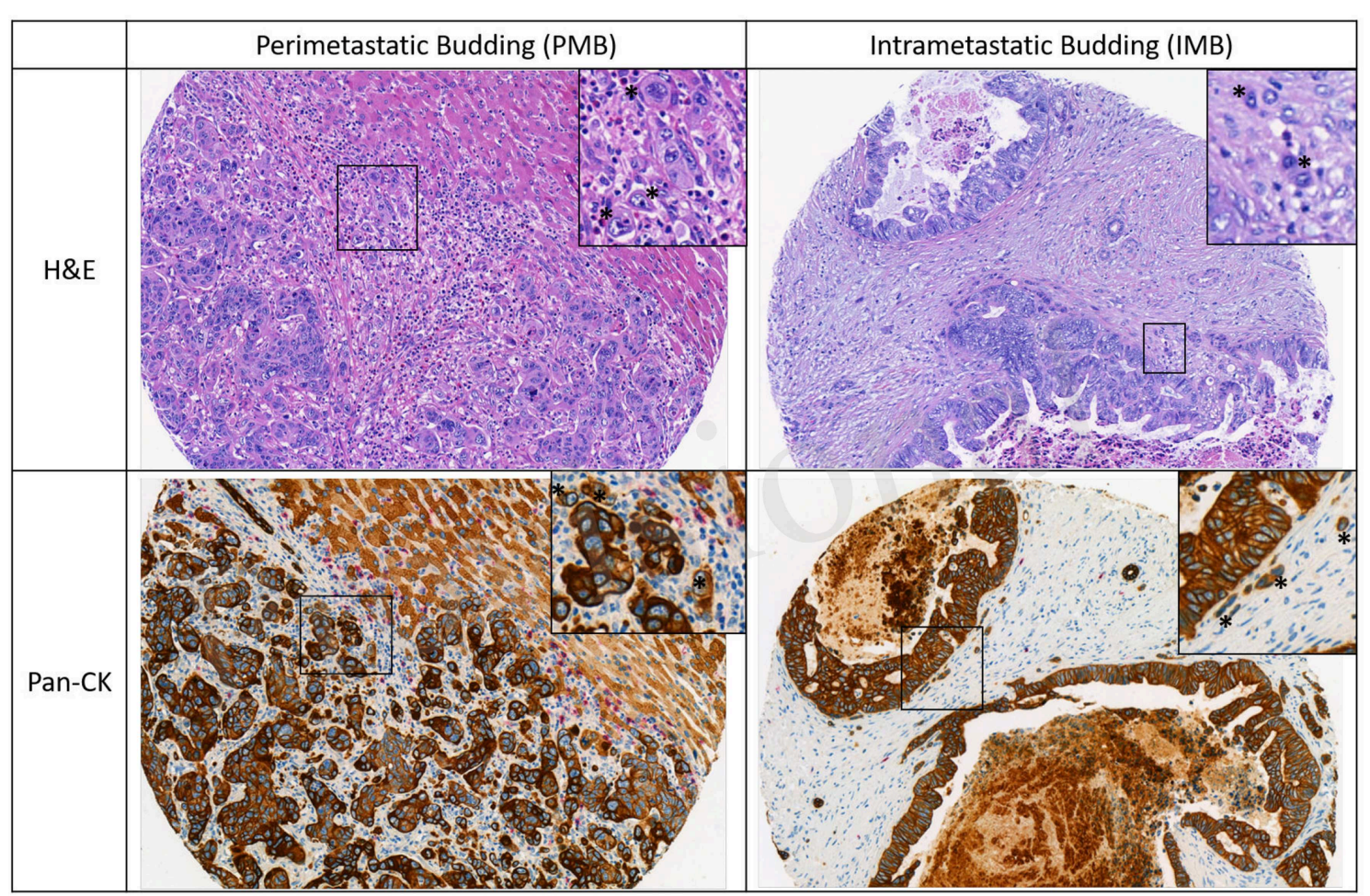

FIGURE 1 | Representative images of perimetastatic and intrametastatic budding on H\&E and Pan-CK staining. Examples of tumor buds are indicated with an asterisk.

TABLE 2 | Differences in the average number of ITB/IMB and PTB/PMB.

\begin{tabular}{llllrll}
\hline Stain & Tissue & Center/Front & No. & Mean & Min & Max \\
\hline H\&E & Primaries & Front & 81 & 8.0 & 0 & 46 \\
H\&E & Primaries & Center & 81 & 6.4 & 0 & 21 \\
Pan-CK & Primaries & Front & 81 & 12.3 & 0 & 53 \\
Pan-CK & Primaries & Center & 81 & 10.2 & 0 & 98 \\
H\&E & Metastases & Front & 74 & 7.4 & 0 & 33 \\
H\&E & Metastases & Center & 74 & 8.9 & 0 & 36 \\
Pan-CK & Metastases & Front & 74 & 9.0 & 0 & 59 \\
Pan-CK & Metastases & Center & 74 & 11.9 & 0 & 71 \\
\hline
\end{tabular}

TABLE 3 | Number of buds in total metastasis and primary with means and test of differences between matched samples.

\begin{tabular}{lcc}
\hline & Mean no. of buds & $\boldsymbol{P}$-value \\
\hline Primaries H\&E & 7.2 & 0.6229 \\
Metastases H\&E & 8.3 & \\
Primaries Pan-CK & 11.3 & 0.0038 \\
Metastases Pan-CK & 7.3 & \\
\hline
\end{tabular}

the process of epithelial mesenchymal transition (EMT) (20-22). As EMT is highly dependent on the tumor microenvironment, one could expect different pathogenetic mechanisms of budding in the liver parenchyma compared to the colorectal wall. The formation of buds might be advantageous in one organ, but obstructive or even destructive at the same time under different circumstances. Consequently, tumor budding in CRLM may differ from its role in primary CRC including the definition of cut offs for therapeutic decision making as well as a prognostic and predictive factor. The significant difference in the mean number of tumor buds between primary tumors and metastases on Pan-CK staining could provide further evidence for this hypothesis.

These assumptions are corroborated by differences in prognosis depending on the observed growth patterns in CRLM. Desmoplastic CRLM are associated with a better prognosis compared with the replacement or pushing type. The replacement type of CRLM demonstrates a close proximity to hepatic sinusoids and has been shown to be non-angiogenic. The desmoplastic and pushing type on the other hand reveal an angiogenic phenotype that might be disadvantageous in a highly vascularized organ like the liver (23-28). A broad rim of collagen could be even more hindering for tumor progression and thus represents an explanation for the observed differences in our study. Tumor cell migration and angiogenesis is an important factor for tumor progression in primary CRC but might be of no or lesser importance in CRLM.

Up to now there are two publications which demonstrate that tumor budding is a prognostic factor in CRLM based on univariate, but not in multi-variate analysis $(18,29)$. This 
TABLE 4 | Correlation of intratumoral and intrametastatic budding (ITB/IMB) with peritumoral and perimetastatic budding (PTB/PMB).

\begin{tabular}{|c|c|c|c|c|c|c|c|c|}
\hline & H\&E РTB & H\&E ITB & Pan-CK PTB & Pan-CK ITB & H\&E PMB & H\&E IMB & Pan-CK PMB & Pan-CK IMB \\
\hline H\&E PTB & 1.0 & & & & & & & \\
\hline H\&E ITB & $0.36^{\star}$ & 1.0 & & & & & & \\
\hline Pan-CK ITB & -0.12 & 0.04 & 0.36 & 1.0 & & & & \\
\hline H\&E PMB & 0.03 & 0.11 & -0.14 & 0.05 & 1.0 & & & \\
\hline Pan-CK IMB & 0.01 & -0.03 & 0.01 & 0.07 & $0.3^{\star}$ & $0.3^{\star}$ & $0.44^{\star}$ & 1.0 \\
\hline
\end{tabular}

Correlation coefficient indicated (r). Asterisk indicates $p<0.05$.

TABLE 5 | Kappa values showing the concordance and percent agreement of bd scores for H\&E and Pan-CK.

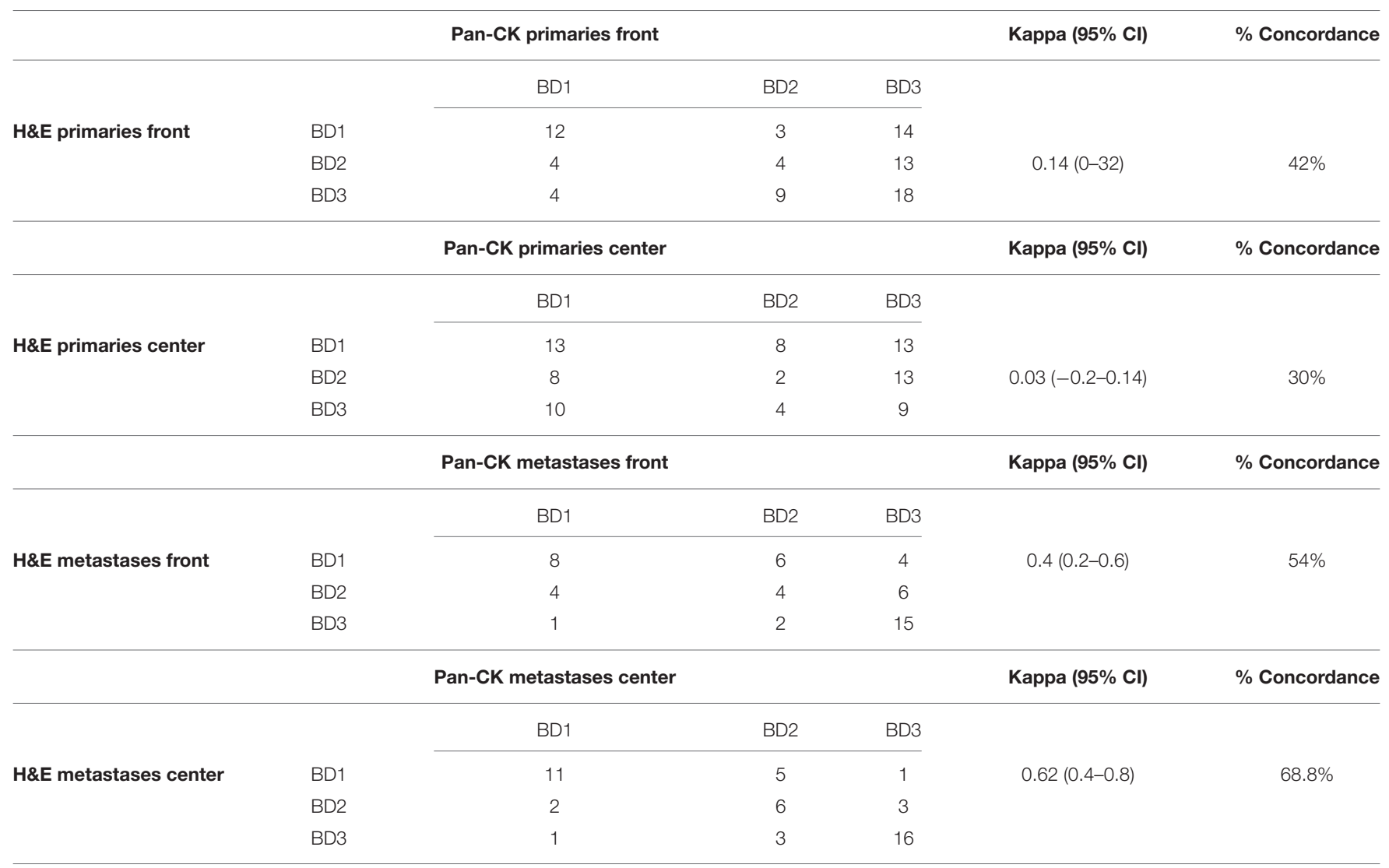

result suggests that other factors such as differing components of the microenvironment, including inflammatory and stromal cells in the colon wall and in the liver may influence tumor budding and therefore have a more important prognostic and/or predictive role.

In primary CRC, pathologists using tumor budding in daily practice know potential pitfalls such as intense peritumoral inflammation, prominent stromal reaction with high numbers of activated macrophages and glandular fragmentation (30). Tumor buds are usually surrounded by desmoplastic stroma. If this is not the case, retraction artifacts or vascular invasion have to be ruled out. Pitfalls associated with the evaluation of tumor budding in CRLM have not been described, yet. Before using tumor budding in CRLM, its methodological challenges needs to be elucidated and discussed. The hepatic microscopic architecture differs significantly from the colon wall. The liver is a highly vascularized organ, consisting of numerous arteries, veins and sinusoids. These architectural differences permits growth patterns other than the ones known from primary tumors $(25,31,32)$. Only the desmoplastic type of CRLM is likely to demonstrate tumor buds. The pushing type, by definition, should not manifest with single tumor cells or clusters at the invasive front. The replacement type can exhibit single cells or tumor cell clusters at the invasive front, but these are more 


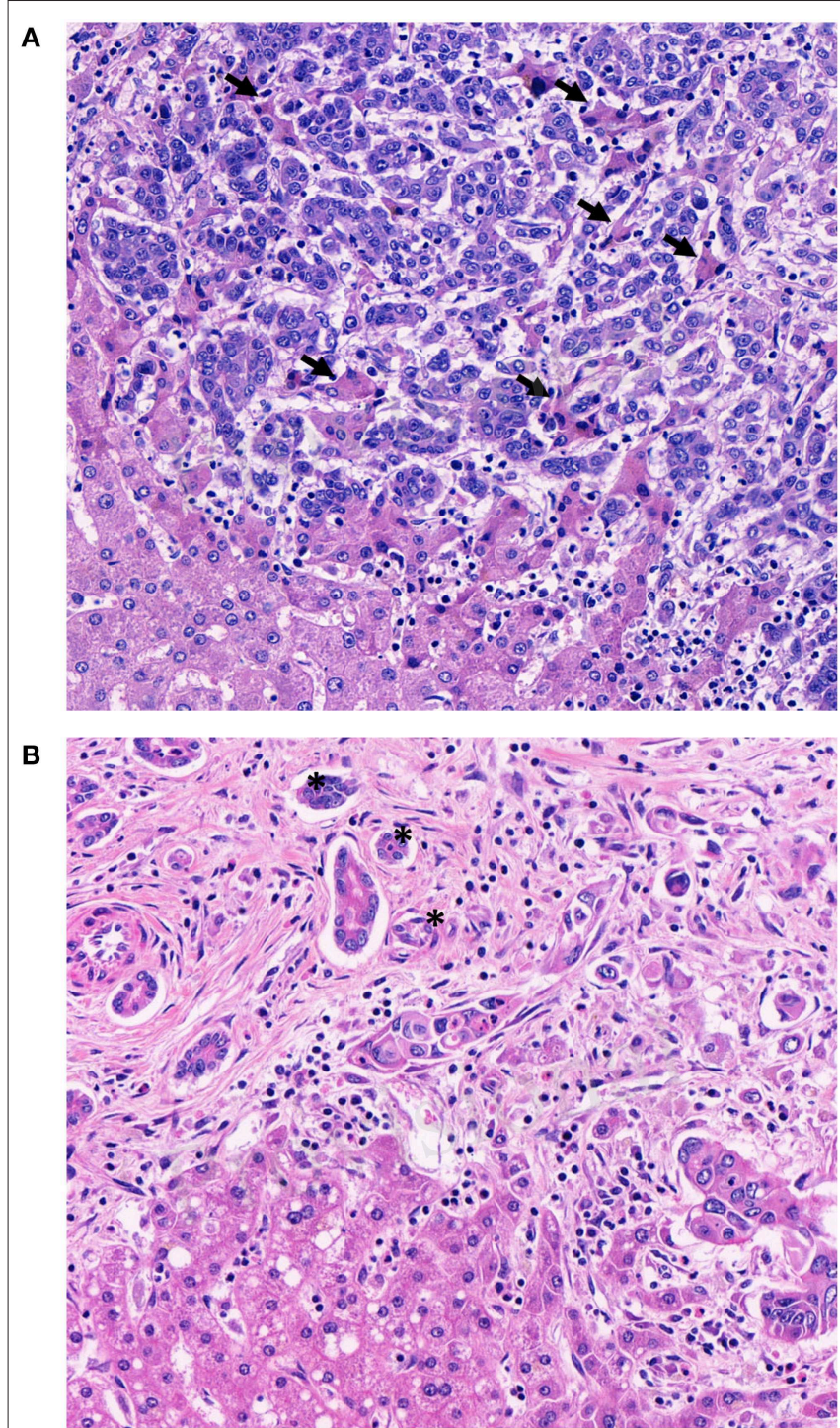

FIGURE 2 | Methodical challenges for tumor budding evaluation in colorectal liver metastases. (A) Single cells or tumor cells clusters in between hepatocellular trabecula without obvious desmoplastic stroma reaction. Examples of hepatocellular trabecula between infiltrating tumor cells are indicated with arrows (B) Tumor buds and ductular reaction with reactive changes in close proximity. Examples of ductular reaction are indicated with an asterisk

likely to represent vascular invasion due to their non-angiogenic phenotype than actual tumor budding (Figure 2A). Second, CRLM are often surrounded by a prominent ductular reaction

\section{REFERENCES}

1. Zlobec I, Lugli A. Tumour budding in colorectal cancer: molecular rationale for clinical translation. Nat Rev Cancer. (2018) 18:203-4. doi: $10.1038 /$ nrc. 2018.1
(Figure 2B) which can mimic tumor budding. Unfortunately, there is no reliable immunohistochemical marker to differentiate tumor buds from ductular reaction. Hence, the pathologist has to rely on morphological features like nuclear-cytoplasmic-ratio, anisokaryosis and hyperchromasia alone to distinguish bile ducts from tumor buds.

In summary, our main goal was to systematically analyze the tumor budding scoring systems in CRLM and therefore focus only on the methodological aspects and not on its predictive and prognostic role. Therefore, we suggest to evaluate tumor budding only in CRLM with desmoplastic stroma reaction on $\mathrm{H} \& \mathrm{E}$ stained slides using the ITBCC method because of two reasons: First, infiltrating tumor cells without surrounding stroma reaction cannot be reliably differentiated from vascular invasion. Second, morphological features to differentiate tumor buds from bile ducts are more easily detected by H\&E than by immunohistochemistry. These aspects should be definitely considered in future large retrospective/prospective trials including tumor budding in stage IV CRC with liver metastases.

\section{DATA AVAILABILITY STATEMENT}

The datasets generated for this study are available on request to the corresponding author.

\section{ETHICS STATEMENT}

Ethical approval was obtained for the use of all tissue and data in this study (KEK\#2017-01803). The studies involving human participants were reviewed and approved by Kantonale Ethikkommission Bern. Written informed consent for participation was not required for this study in accordance with the national legislation and the institutional requirements.

\section{AUTHOR CONTRIBUTIONS}

$\mathrm{AB}$ and CS: writing and editing of the original draft. HD, $\mathrm{GB}$, and IZ: reviewing and editing. AL: writing, reviewing, and editing.

\section{FUNDING}

The authors declare that this study received personal funding from the Nuovo-Soldati foundation and funding of consumables from the Stiftung KrebsHilfe and the Stiftung zur Krebsbekämpfung. The funders had no role in study design, data collection and analysis, decision to publish, or preparation of the manuscript.

2. James D. Brierley MKG, Christian Wittekind. TNM Classification of Malignant Tumours. 8th ed. (2017). doi: 10.1002/9780471420194. tnmc26.pub3

3. Ueno H, Ishiguro M, Nakatani E, Ishikawa T, Uetake H, Matsuda C, et al. Prospective multicenter study on the prognostic and predictive impact of 
tumor budding in stage II colon cancer: results from the SACURA trial. J Clin Oncol. (2019) 37:1886-94. doi: 10.1200/JCO.18.02059

4. Dawson H, Galuppini F, Trager P, Berger MD, Studer P, Brugger L, et al. Validation of the International Tumor Budding Consensus Conference 2016 recommendations on tumor budding in stage I-IV colorectal cancer. Hum Pathol. (2019) 85:145-51. doi: 10.1016/j.humpath.2018.10.023

5. Yasue C, Chino A, Takamatsu M, Namikawa K, Ide D, Saito S, et al. Pathological risk factors and predictive endoscopic factors for lymph node metastasis of T1 colorectal cancer: a single-center study of 846 lesions. J Gastroenterol. (2019) 54:708-17. doi: 10.1007/s00535-01901564-y

6. Oh BY, Park YA, Huh JW, Yun SH, Kim HC, Chun HK, et al. prognostic impact of tumor-budding grade in stages 1-3 colon cancer: a retrospective cohort study. Ann Surg Oncol. (2018) 25:204-11. doi: 10.1245/s10434-017-6135-5

7. Landau MA, Zhu B, Akwuole FN, Pai RK. Histopathological predictors of recurrence in stage III colon cancer: reappraisal of tumor deposits and tumor budding using AJCC8 criteria. Int J Surg Pathol. (2019) 27:147-58. doi: $10.1177 / 1066896918787275$

8. Lee VWK, Chan KF. Tumor budding and poorly-differentiated cluster in prognostication in Stage II colon cancer. Pathol Res Pract. (2018) 214:402-7. doi: 10.1016/j.prp.2017.12.019

9. Slik K, Blom S, Turkki R, Valimaki K, Kurki S, Mustonen H, et al. Combined epithelial marker analysis of tumour budding in stage II colorectal cancer. $J$ Pathol Clin Res. (2019) 5:63-78. doi: 10.1002/cjp2.119

10. Trinh A, Ladrach C, Dawson HE, Ten Hoorn S, Kuppen PJK, Reimers MS, et al. Tumour budding is associated with the mesenchymal colon cancer subtype and RAS/RAF mutations: a study of 1320 colorectal cancers with Consensus Molecular Subgroup (CMS) data. Br J Cancer. (2018) 119:1244-51. doi: $10.1038 /$ s41416-018-0230-7

11. Lino-Silva LS, Gamboa-Dominguez A, Zuniga-Tamayo D, SalcedoHernandez RA, Cetina L, Cantu-de-Leon D. Mismatch repair protein expression and intratumoral budding in rectal cancer are associated with an increased pathological complete response to preoperative chemoradiotherapy: a case-control study. World J Clin Oncol. (2018) 9:133-9. doi: 10.5306/wjco.v9.i7.133

12. Tsai JH, Jeng YM, Yuan CT, Lin YL, Cheng ML, Liau JY. Traditional serrated pathway-associated colorectal carcinoma: morphologic reappraisal of serrated morphology, tumor budding, and identification of frequent PTEN alterations. Am J Surg Pathol. (2019) 43:1042-51. doi: 10.1097/PAS.0000000000001274

13. Lugli A, Kirsch R, Ajioka Y, Bosman F, Cathomas G, Dawson H, et al. Recommendations for reporting tumor budding in colorectal cancer based on the International Tumor Budding Consensus Conference (ITBCC) 2016. Modern Pathol. (2017) 30:1299-311. doi: 10.1038/modpathol. 2017.46

14. Lugli A, Vlajnic T, Giger O, Karamitopoulou E, Patsouris ES, Peros G, et al. Intratumoral budding as a potential parameter of tumor progression in mismatch repair-proficient and mismatch repair-deficient colorectal cancer patients. Hum Pathol. (2011) 42:1833-40. doi: 10.1016/j.humpath.2011.02.010

15. Morodomi $\mathrm{T}$, Isomoto $\mathrm{H}$, Shirouzu $\mathrm{K}$, Kakegawa $\mathrm{K}$, Irie $\mathrm{K}$, Morimatsu M. An index for estimating the probability of lymph node metastasis in rectal cancers. Lymph node metastasis and the histopathology of actively invasive regions of cancer. Cancer. (1989) 63:539-43. doi: 10.1002/10970142(19890201)63:3<539::AID-CNCR2820630323>3.0.CO;2-S

16. Zlobec I, Hadrich M, Dawson H, Koelzer VH, Borner M, Mallaev M, et al. Intratumoural budding (ITB) in preoperative biopsies predicts the presence of lymph node and distant metastases in colon and rectal cancer patients. $\mathrm{Br} \mathrm{J}$ Cancer. (2014) 110:1008-13. doi: 10.1038/bjc.2013.797

17. Giger OT, Comtesse SC, Lugli A, Zlobec I, Kurrer MO. Intra-tumoral budding in preoperative biopsy specimens predicts lymph node and distant metastasis in patients with colorectal cancer. Modern Pathol. (2012) 25:104853. doi: 10.1038/modpathol.2012.56

18. Fonseca GM, de Mello ES, Faraj SF, Kruger JAP, Coelho FF, Jeismann VB, et al. Prognostic significance of poorly differentiated clusters and tumor budding in colorectal liver metastases. J Surg Oncol. (2018) 117:1364-75. doi: $10.1002 /$ jso. 25017
19. Lytle NK, Ferguson LP, Rajbhandari N, Gilroy K, Fox RG, Deshpande A, et al. A multiscale map of the stem cell state in pancreatic adenocarcinoma. Cell. (2019) 177:572-86.e22. doi: 10.1016/j.cell.2019.03.010

20. Zlobec I, Lugli A. Epithelial mesenchymal transition and tumor budding in aggressive colorectal cancer: tumor budding as oncotarget. Oncotarget. (2010) 1:651-61. doi: 10.18632/oncotarget.199

21. Brabletz T, Hlubek F, Spaderna S, Schmalhofer O, Hiendlmeyer E, Jung A, et al. Invasion and metastasis in colorectal cancer: epithelial-mesenchymal transition, mesenchymal-epithelial transition, stem cells and beta-catenin. Cells Tissues Organs. (2005) 179:56-65. doi: 10.1159/000084509

22. Grigore AD, Jolly MK, Jia D, Farach-Carson MC, Levine H. Tumor budding: the name is EMT. Partial EMT. J Clin Med. (2016) 5:51. doi: $10.3390 / \mathrm{jcm} 5050051$

23. Van den Eynden GG, van Dam PJ, Stroobants S, Dirix L, Vermeulen P, Liver Metastasis Research N. Histopathological evaluation of resected colorectal cancer liver metastases: what should be done? Histopathology. (2014) 64:3156. doi: 10.1111/his.12259

24. Van den Eynden GG, Majeed AW, Illemann M, Vermeulen PB, Bird NC, Hoyer-Hansen G, et al. The multifaceted role of the microenvironment in liver metastasis: biology and clinical implications. Cancer Res. (2013) 73:2031-43. doi: 10.1158/0008-5472.CAN-12-3931

25. Van den Eynden GG, Bird NC, Majeed AW, Van Laere S, Dirix LY, Vermeulen PB. The histological growth pattern of colorectal cancer liver metastases has prognostic value. Clin Exp Metastasis. (2012) 29:541-9. doi: 10.1007/s10585-012-9469-1

26. Lazaris A, Amri A, Petrillo SK, Zoroquiain P, Ibrahim N, Salman A, et al. Vascularization of colorectal carcinoma liver metastasis: insight into stratification of patients for anti-angiogenic therapies. J Pathol Clin Res. (2018) 4:184-92. doi: $10.1002 / \mathrm{cjp} 2.100$

27. Frentzas S, Simoneau E, Bridgeman VL, Vermeulen PB, Foo S, Kostaras $\mathrm{E}$, et al. Vessel co-option mediates resistance to anti-angiogenic therapy in liver metastases. Nat Med. (2016) 22:1294-302. doi: 10.1038/ nm. 4197

28. Eefsen RL, Vermeulen PB, Christensen IJ, Laerum OD, Mogensen $\mathrm{MB}$, Rolff HC, et al. Growth pattern of colorectal liver metastasis as a marker of recurrence risk. Clin Exp Metastasis. (2015) 32:369-81. doi: 10.1007/s10585-015-9715-4

29. Yonemura K, Kajiwara Y, Ao T, Mochizuki S, Shinto E, Okamoto K, et al. Prognostic value of poorly differentiated clusters in liver metastatic lesions of colorectal carcinoma. Am $J$ Surg Pathol. (2019) 43:1341-8. doi: 10.1097/PAS.000000000 0001329

30. Mitrovic B, Schaeffer DF, Riddell RH, Kirsch R. Tumor budding in colorectal carcinoma: time to take notice. Modern Pathol. (2012) 25:1315-25. doi: 10.1038/modpathol.2012.94

31. Hoppener DJ, Nierop PMH, Herpel E, Rahbari NN, Doukas M, Vermeulen $\mathrm{PB}$, et al. Histopathological growth patterns of colorectal liver metastasis exhibit little heterogeneity and can be determined with a high diagnostic accuracy. Clin Exp Metastasis. (2019) 36:311-9. doi: 10.1007/s10585-019-09975-0

32. van Dam PJ, van der Stok EP, Teuwen LA, Van den Eynden GG, Illemann $\mathrm{M}$, Frentzas $\mathrm{S}$, et al. International consensus guidelines for scoring the histopathological growth patterns of liver metastasis. Br J Cancer. (2017) 117:1427-41. doi: 10.1038/bjc.2017.334

Conflict of Interest: The authors declare that the research was conducted in the absence of any commercial or financial relationships that could be construed as a potential conflict of interest.

Copyright (c) 2019 Blank, Schenker, Dawson, Beldi, Zlobec and Lugli. This is an open-access article distributed under the terms of the Creative Commons Attribution License (CC BY). The use, distribution or reproduction in other forums is permitted, provided the original author(s) and the copyright owner(s) are credited and that the original publication in this journal is cited, in accordance with accepted academic practice. No use, distribution or reproduction is permitted which does not comply with these terms. 